\title{
Paraneoplastic cerebellar ataxia associated with anti-Hu antibodies and benign ganglioneuroma
}

\author{
Roberto Fancellu, MDa \\ Elena Corsini, BSc ${ }^{b}$ \\ Gaetano Bernardi, MD \\ Paolo Buzzo, MDc \\ Maria Luisa Ferrari, MDa \\ Eleonora Lamantea, BSc ${ }^{d}$ \\ Alberto Garaventa, MD \\ Mauro Truini, MD' \\ Sandro Salvarani, MDa
}

\begin{abstract}
a Unit of Neurology, Villa Scassi Hospital ASL3, Genoa, Italy

' Unit of Laboratory Analysis, Fondazione IRCCS

Istituto Neurologico Carlo Besta, Milan, Italy

- Unit of General Medicine, Villa Scassi Hospital

ASL3, Genoa, Italy

dUnit of Molecular Neurogenetics, Fondazione

IRCCS Istituto Neurologico Carlo Besta, Milan, Italy

e Department of Pediatric Hematology/Oncology,

Giannina Gaslini Institute, Genoa, Italy

$\mathrm{t}$ Unit of Pathological Anatomy and Cytohistology,

IRCCS S. Martino University Hospital - IST National

Institute for Cancer Research, Genoa, Italy
\end{abstract}

Correspondence to: Roberto Fancellu

E-mail: roberto.fancellu@hsanmartino.it

\section{Summary}

We describe a case of cerebellar ataxia associated with anti-Hu antibodies and benign ganglioneuroma. A 28-year-old woman developed progressive ataxia with hyporeflexia at the age of 19. Brain MRI showed progressive cerebellar atrophy. Neurophysiological studies, screening of immune-mediated ataxias, oncological markers, vitamin $\mathrm{E}$ and genetic tests for spinocerebellar ataxia types 1,2,3, Friedreich ataxia and POLG1 were negative. Anti-Hu antibodies were positive in Western blot and indirect immunofluorescence $(1: 640)$. Total-body computed tomography revealed a mediastinum mass; the histological diagnosis was maturing ganglioneuroma. Immunohistochemistry showed a mild reaction between the tumor and the patient's serum, and no reaction between the tumor and control serum. After surgery, serum
anti-Hu titer decreased, while ataxic symptoms initially worsened and then stabilized. Ganglioneuroma is a benign tumor, usually derived from the maturation of a neuroblastoma. The benign histology and the presence of anti-Hu antibodies could be related to the positive oncological prognosis and to the slow clinical course mimicking a degenerative ataxia.

KEY WORDS: anti-Hu antibodies, ganglioneuroma, immune-mediated ataxia, neuroblastoma, paraneoplastic syndrome.

\section{Introduction}

We describe the case of a woman presenting paraneoplastic cerebellar degeneration (PCD) associated with anti-Hu antibodies and ganglioneuroma, a neuroblastic tumor with a benign histology. PCD is a syndrome characterized by progressive cerebellar ataxia, related to the presence of a tumor, and possibly associated with onconeural antibodies (Graus and Dalmau, 2012). In pediatric patients, PCD may present with ocular and limb involuntary movements (opsoclonusmyoclonus-ataxia); in several affected children, the tumor responsible was found to be a neuroblastoma (Gambini et al., 2003).

\section{Case report}

The patient here described is a 28-year-old woman who developed progressive ataxia at the age of 19 . She reported a history of scoliosis from the age of 4 years and fluctuating peripheral hearing impairment from the age of 10 . At 14 years, brain magnetic resonance imaging (MRI) was normal, while at 23 years a control MRI study showed cerebellar atrophy without signal alterations. At the age of 25 years, neurological examination revealed moderate cerebellar gait, mild limb dysmetria, hyporeflexia in all four limbs, gazeevoked horizontal nystagmus, and a mildly positive Romberg test; it was calculated that the patient would have scored 8/40 on the Scale for Assessment and Rating of Ataxia (SARA) (Schmitz-Hübsch et al., 2006). She never reported opsoclonus-myoclonus. Several diagnostic investigations gave normal findings, including complete neurophysiological studies, screening of immune-mediated ataxias, antibodies associated with celiac disease, oncological markers, alpha-fetoprotein, 


\section{R. Fancellu et al.}

vitamin E, acanthocytes, and genetic tests for spinocerebellar ataxia types 1,2,3, and Friedreich ataxia; molecular analysis of the POLG1 gene detected the known heterozygous mutation c803G>C (pG268A), while multiplex ligation-dependent probe amplification was negative. Anti-nuclear antibodies were found at variable titers, from negative to 1:640. Anti-Hu antibodies were positive in Western blot and indirect immunofluorescence performed on rat cerebellar slices (1:640). Two total-body positron-emission tomography scans showed mild non-specific tracer accumulations. Total-body computed tomography (CT) scanning revealed, in the left superoposterior mediastinum, an expansive lesion with mild peripheral enhancement, and without compression or infiltration of adjacent structures or lymph node enlargement (Fig. 1, panels A and B). This mass was completely excised. Pathological examination, performed following the International Neuroblastoma Pathology Committee (INPC) recommendations (Shimada et al., 1999), described a $8 \times 3 \times 4 \mathrm{~cm}$ neuromatous proliferative mass, with a smooth surface, composed of spindle cells arranged in variably oriented bundles, distributed in a focally myxoid fibrovascular stroma; in this background, scattered neuron-specific-enolase-positive ganglion cells were observed, in some cases with prominent nucleoli or with two nuclei. Neuromatous cells expressed S100 protein. Neither defined nodules of neuroblasts nor necrosis, calcifications or inflammatory infiltrates were observed (Fig. 1, panel C). The histological diagnosis was ganglioneuroma, maturing subtype (Shimada et al., 1999).

To better characterize the possible relationship between anti-Hu antibodies and ganglioneuroma, we performed immunohistochemical assay by exposing tumor sections to the patient's serum or to control serum; the tumor cells mildly reacted with the patient's serum, while no immunoreaction was observed with the control serum (Fig. 1, panels D and E). After surgery, serum anti-Hu titers decreased (Table I), while gait ataxia and
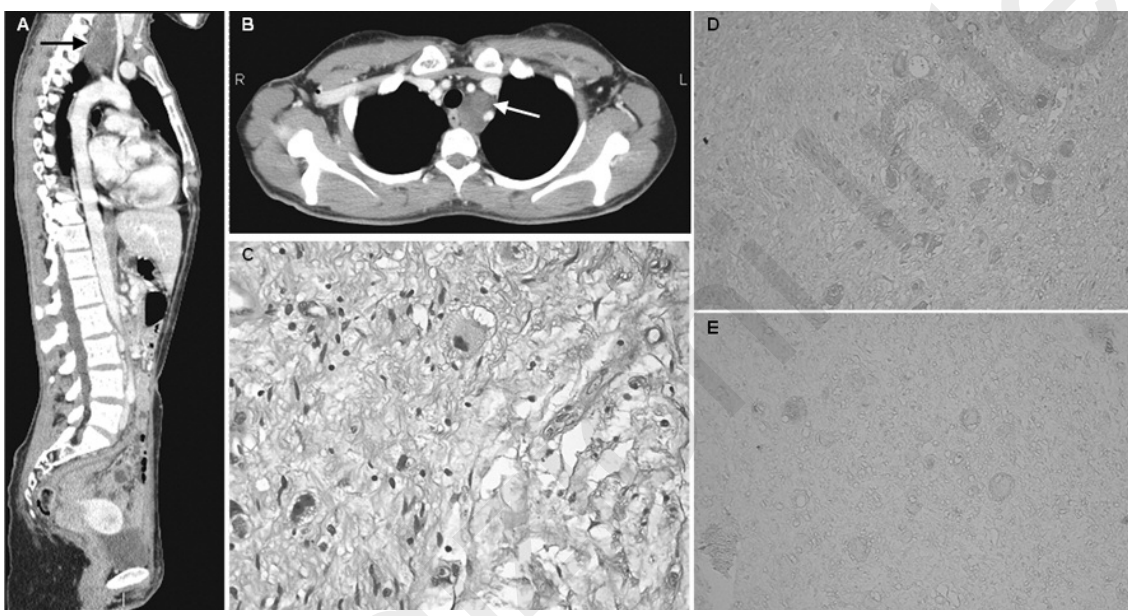

Figure 1 - Radiological, pathological and immunohistochemical features of diagnosed ganglioneuroma.

A and B: Whole-body CT scan showing an expansive lesion in left superoposterior mediastinum (arrows).

C: Hematoxylin-eosin staining of resected ganglioneuroma (original magnification $\times 40$ ).

$\mathrm{D}$ and $\mathrm{E}$ : Patient's ganglioneuroma sections exposed to patient's serum (diluted 1:20, D) or control serum (diluted 1:20, E). (original magnification $\mathrm{x} 20$ ).

Table I - Anti-Hu titers and therapeutic interventions during disease course.

\begin{tabular}{llll}
\hline $\begin{array}{l}\text { Time from tumor } \\
\text { diagnosis (months) }\end{array}$ & $\begin{array}{l}\text { Immunohistochemistry } \\
\text { (serum anti-Hu titer) }\end{array}$ & Immunoblot positivity & Treatments \\
\hline Pre-surgery & Positive 1:640 & $3+$ & - \\
-1 & - & - & Intravenous immunoglobulins \\
0 & - & - & Surgery \\
+4 & Positive $1: 160$ & $2+$ & - \\
+6 & Positive $1: 320$ & $1+$ & - \\
+8 & Positive $1: 80$ & $<1+$ & Intravenous immunoglobulins \\
+11 & Positive $1: 80$ & $1+$ & - \\
+13 & Positive $1: 80$ & $1+$ & - \\
+15 & - & - & Intravenous steroids \\
+16 & Positive $1: 80$ & $1+$ & Intravenous steroids \\
+17 & - & - & Plasma exchange \\
+18 & Positive $1: 40$ & $1+$ & - \\
+20 & - & - & Rituximab \\
+21 & Positive $1: 160$ & $1+$ & - \\
+24 & Positive $1: 160$ & $1+$ & - \\
\hline
\end{tabular}


disequilibrium first worsened and then stabilized; the patient reached a SARA score of 13/40. Total-body CT, cervical MRI, serum lactate dehydrogenase and urinary vanil-mandelic and homovanillic acids were normal at 12 and 24 months after surgery, brain MRI was unchanged at six and 24 months. Whole-body metaiodobenzylguanidine scintigraphy was negative.

The patient was treated with physical therapy, recurrent oral steroids, two courses of intravenous immunoglobulins, two courses of intravenous steroids, five courses of plasma exchange, and two intravenous administrations of rituximab $\left(375 \mathrm{mg} / \mathrm{m}^{2}\right.$, separated by a two-week interval), without significant effects (Table I).

\section{Discussion}

This case represents the first association of cerebellar ataxia, anti-Hu antibodies and maturing ganglioneuroma. Anti-Hu antibodies are defined as "well-characterized" onconeural antibodies, highly predicting the presence of a tumor (Graus and Dalmau, 2012; Dalmau et al., 1995). They have already been described in patients with opsoclonus-myoclonus-ataxia affected by neuroblastoma (Dalmau et al., 1995; Salmaggi et al., 1997; Jarius et al., 2009), but not in ataxic patients with ganglioneuroblastoma or ganglioneuroma. Moreover, the $\mathrm{Hu}$ antigen is expressed in neuroblastoma cell lines and in a proportion of neuroblastomas (Dalmau et al., 1995).

The neuroblastic tumors lie along a histological continuum ranging from benign ganglioneuroma through intermediate ganglioneuroblastoma to malignant neuroblastoma, with transitional subtypes (Shimada et al., 1999). In our case, the presence of "well-characterized" antibodies (anti-Hu) and neurological syndrome (PCD) allows the paraneoplastic syndrome to be diagnosed as "definite" in accordance with the diagnostic criteria of Graus and Dalmau (2012). A possible pathogenetic role of the ganglioneuroma is suggested by the finding of an immunohistochemical reaction between tumor antigens and the patient's serum, and by the reduction of the anti-Hu titer after tumor resection; the persistence of anti-Hu antibodies after surgical excision has already been described by others (Jarius et al., 2009). We did not observe a significant clinical improvement after surgical and pharmacological treatment. In paraneoplastic syndromes, in fact, the brain damage is due principally to cytotoxic $T$ cells, while anti-Hu antibodies, as well as other antibodies against intracellular antigens, may be an epiphenomenon; this may explain the persistence of anti-Hu antibodies after surgical or pharmacological therapy. Moreover, these data may be related to the variable response to the treatments, as we observed in our case.

Our patient, however, has some peculiarities, particularly an older age at onset and a slower course of ataxia compared with classical PCD associated with neuroblastoma. It has been reported that neuroblastoma, usually proliferating and aggressive, may show involution or spontaneous regression (Shimada et al.,
1999; Hero et al., 2008); a very uncommon evolution of neuroblastoma is spontaneous and pathologicallydocumented maturation into benign ganglioneuroma even without treatment (Rothenberg et al., 2009; Cushing and Wolbach, 1927; Fox et al., 1959; Haas et al., 1988). Interestingly, the first report of spontaneous maturation of neuroblastoma to ganglioneuroma is also the first unwitting description of opsoclonusmyoclonus-ataxia syndrome associated with neuroblastoma (Cushing and Wolbach, 1927). Moreover, a favorable prognosis has been described in some malignant tumors (such as neuroblastoma and small cell lung cancer) when associated with paraneoplastic syndromes (Dalmau et al., 1995; Jarius et al., 2009; Rothenberg et al., 2009; Altman and Baehner, 1976). It could thus be hypothesized that our patient at an early age developed a silent neuroblastoma which determined the synthesis of anti-Hu antibodies and matured to ganglioneuroma; these two conditions could explain the positive oncological prognosis and the slow clinical course mimicking a degenerative cerebellar ataxia.

\section{Acknowledgments}

We wish to thank Drs Omar Racchi, Paola Tognetti, Daria Schettini, and Giovanni Battista Ratto for their expert contributions.

\section{References}

Altman AJ, Baehner RL (1976). Favorable prognosis for survival in children with coincident opso-myoclonus and neuroblastoma. Cancer 37:846-852.

Cushing H, Wolbach SB (1927). The transformation of a malignant paravertebral sympathicoblastoma into a benign ganglioneuroma. Am J Pathol 3:203-216.

Dalmau J, Graus F, Cheung NK, et al (1995). Major histocompatibility proteins, anti-Hu antibodies, and paraneoplastic encephalomyelitis in neuroblastoma and small cell lung cancer. Cancer 75:99-109.

Fox F, Davidson J, Thomas LB (1959). Maturation of sympathicoblastoma into ganglioneuroblastoma: report of 2 patients with 20- and 46-year survivals respectively. Cancer 12:108-116.

Gambini C, Conte M, Bernini G, et al (2003). Neuroblastic tumors associated with opsoclonus-myoclonus syndrome: histological, immunohistochemical and molecular features of 15 Italian cases. Virchows Arch 442:555-562.

Graus F, Dalmau J (2012). Paraneoplastic neurological syndromes. Curr Opin Neurol 25:795-801.

Haas D, Ablin AR, Miller C, et al (1988). Complete pathologic maturation and regression of stage IVS neuroblastoma without treatment. Cancer 62:818-825.

Hero B, Simon T, Spitz R, et al (2008). Localized infant neuroblastomas often show spontaneous regression: results of the prospective trials NB95-S and NB97. J Clin Oncol 26:1504-1510.

Jarius S, Arnold S, Linke R, et al (2009). Long term survival in anti-Hu associated adult neuroblastoma. J Neurol Sci 284:205-208.

Rothenberg AB, Berdon WE, D'Angio GJ, et al (2009). The association between neuroblastoma and opsoclonus- 
myoclonus syndrome: a historical review. Pediatr Radiol 39:723-726.

Salmaggi A, Nemni R, Pozzi A, et al (1997). Antineuronal antibody in a patient with neuroblastoma and opsoclonusmyoclonus-ataxia: a case report. Tumori 83:709-711.

Schmitz-Hübsch T, du Montcel ST, Baliko L, et al (2006). Scale for the assessment and rating of ataxia: development of a new clinical scale. Neurology 66:1717-1720.

Shimada H, Ambros IM, Dehner LP, et al (1999). Terminology and morphologic criteria of neuroblastic tumors: recommendations by the International Neuroblastoma Pathology Committee. Cancer 86:349-363. 